\title{
Toxina botulínica A e intervenção fisioterapêutica no tratamento do ombro doloroso pós acidente vascular cerebral: relato de caso
}

\section{Botulinum toxin A and physiotherapy intervention in the treatment of the painful shoulder after stroke: case report}

\author{
Karen Lúcia de Araújo Freitas Moreira'; Cristina Katya Dantas Torres²; \\ Mayara Dinamine França Dantas²; Suellen M. Marinho dos Santos Andrade ${ }^{3}$
}

\section{Resumo}

A síndrome do ombro doloroso é considerada um achado comum em pacientes que sofreram acidente vascular cerebral. Várias modalidades terapêuticas são apontadas para melhoria do quadro clínico dos pacientes. Neste relato, será apresentado um caso de síndrome do ombro doloroso, cujo tratamento consistiu no emprego de toxina botulínica A associada à fisioterapia. Após quatro meses de tratamento, houve melhorias significativas quanto à dor, espasticidade, amplitude de movimento e qualidade de vida, e isso sugere ser essa associação terapêutica uma ferramenta eficaz contra os efeitos incapacitantes relacionados a esta síndrome.

Palavras-chave: Síndrome do ombro doloroso. Acidente vascular cerebral.Toxina botulínica A.Fisioterapia.

\begin{abstract}
The syndrome of the painful shoulder is considered a common finding in patients who suffered stroke. Several therapeutic modalities are suggested for the improvement of the patients' clinical condition. In this report, a case of syndrome of the painful shoulder will be discussed, whose treatment consisted of the use of botulinum toxin A associated to physiotherapy. After a four-month treatment, significant improvements occurred concerning pain, spasticity, range of motion and quality of life, suggesting that this therapeutic association is an efficient tool against the incapacitating effects related to this syndrome. Key words: Syndrome of the painful shoulder. Stroke. Botulinum toxin A. Physiotherapy,.
\end{abstract}

\footnotetext{
1 Mestre em Engenharia Biomédica, Professora Titular, Departamento de Fisioterapia, Universidade Federal da Paraíba.E-mail: karenfisio2004@yahoo.com.br.

${ }^{2}$ Quinto ano de Fisioterapia, Universidade Federal da Paraíba

3 Quinto ano de Fisioterapia, Pesquisadora PIVIC/CNPQ, Universidade Federal da Paraíba
} 


\section{Introdução}

As doenças cerebrovasculares são a terceira causa de óbito em países desenvolvidos (HOLANDA et al., 2006) e estatísticas demonstram que têm alta incidência e são a primeira causa de morte no Brasil (FERNANDES; CARVALHO; PRADO, 2006).

A interrupção aguda do fluxo sangüíneo de alguma parte do cérebro origina o que se chama de isquemia cerebral. Quando isso acontece por um determinado período de tempo, podem ocorrer lesões, e este processo caracteriza o acidente vascular cerebral (AVC), conforme aponta Machado (2000).

A espasticidade é uma das principais características das lesões do sistema nervoso central (SNC) por acidente vascular cerebral, e acomete os tratos corticoespinais e a via justapiramidal (PONTES et al., 2000). Pode ser definida como uma desordem motora caracterizada pelo aumento do reflexo tônico de estiramento,"velocidade-dependente", com exacerbação dos reflexos tendinosos, resultado do aumento reflexo estiramento, como um dos componentes da síndrome do neurônio motor superior (MEYTHALER, 2001). Essa desordem agrava-se devido ao aumento de citocinas, choques emotivos e estímulos nociceptivos ou sensitivos de outra natureza. Além disso, ela prejudica o funcionamento dos sistemas motores, limita a amplitude de movimento articular e provoca dores, e pode causar aumento da incapacidade funcional, pois afeta o posicionamento confortável do indivíduo e o prejudica nas tarefas de vida diária, tais como alimentação, locomoção, transferências e higiene pessoal, chegando a perturbar-lhe o sono (MUSSE et al., 2002).

Dentre as complicações da espasticidade, a síndrome do ombro doloroso do hemiplégico (ODH) representa um dos principais problemas. A dor é incapacitante e atrapalha a qualidade de vida do paciente, além de criar dificuldades para reabilitação fisioterapêutica (FRAGOSO; ARAÚJO; VELOSO, 2004). Suas possíveis causas estão nos mecanismos de desalinhamento do ombro, movimentações incorretas e imobilidades (HORN et al., 2003). Esta síndrome pode ser considerada comum em pacientes que sofreram AVC, cuja incidência é de 70 a $84 \%$ dos casos.O quadro caracteriza-se por dor no ombro e perda progressiva da amplitude de movimento articular, e, nos casos mais graves, essa dor pode apresentar-se até em repouso (O'SULLIVAN; SCHMITZ, 2004).

O mecanismo exato de dor pós- AVC não é claramente conhecido (SILVA; RIBERTO; BATTISTELL, 2000), porém há consenso quanto aos componentes essenciais da terapêutica escolhida para a redução da inabilidade física e melhoria funcional para a redução da inabilidade física e melhoria funcional (JÜRGEL et al., 2005), como o aumento da amplitude de movimento (ADM), da força ativa dos músculos do ombro e da diminuição da dor. Voltados a estes objetivos, a eletroestimulação, cinesioterapia e o bloqueio químico com toxina botulínica A (TBA) constituem-se em ferramentas eficazes para reabilitação dos pacientes envolvidos (SENEVIRATNE; THEN; REIMER, 2005; YELNIK; COLLE; BONAN, 2003; LISINSKI; GRABARCZYK, 2005).

Portanto, este estudo teve como finalidade avaliar a eficácia da toxina botulínica A aliada à intervenção fisioterapêutica, no tratamento do ombro doloroso de uma paciente pós- AVC.

\section{Causuística e métodos}

Relata-se o caso de uma paciente, do sexo feminino, 49 anos, com seqüelas de acidente vascular cerebral, do tipo isquêmico intraparenquimatoso, comprovado por exame de tomografia computadorizada, atendida na Clínica Escola de Fisioterapia, da Universidade Federal da Paraíba.

Bimestralmente, realizaram-se avaliações cinesiofuncionais, que foram comparadas ao final da pesquisa. Essas avaliações compreendiam dados pessoais, diagnóstico, afecções concomitantes, medicamentos, história da doença e queixas apresentadas pela paciente. Observaram-se também 
as funções cardiovasculares e respiratórias, alterações de trofismo, encurtamentos musculares, sensibilidade e reflexos medulares; todos se apresentaram sem anormalidades. Foi mensurado o tônus muscular, segundo a Escala de Ashworth (PÒO et al., 2003), o número de espasmos, conforme Escala de Espasmos de Penn (ACEVEDO et al., 2000) e a intensidade de dor, segundo a Escala Analógica Visual (LUTTI et al., 2002). A goniometria realizada apresentou os seguintes graus de amplitude de movimento (ADM) para articulação do ombro afetado: flexão $\left(0^{\circ}\right)$, hiperextensão $\left(0^{\circ}\right)$, abdução $\left(0^{\circ}\right)$ e extensão de cotovelo $\left(0^{\circ}\right)$. À avaliação inicial, a paciente mostrava-se eupnéica, normocárdica, normotensa, normocorada, consciente, orientada, sem nenhuma alteração visual, auditiva ou distúrbio na comunicação e nas capacidades perceptivas. Apresentava hemiplegia esquerda, hipertonia grau 3, no músculo bíceps braquial, e grau 5, nos músculos flexores do punho, flexores dos dedos e adutor do polegar do membro superior esquerdo (MSE), segundo escala de Ashworth. Conforme a escala de Penn, apresentou mais de um e menos de dez espasmos espontâneos por hora no MSE (4 espasmos). Associado ao quadro clínico, o quadro álgico impossibilitava-a de realizar qualquer movimento na articulação gleno-umeral, atingindo escore 9, segundo a Escala Analógica Visual.

Quanto ao protocolo de atendimento, foram realizadas três sessões semanais, com duração de 60 minutos cada, sendo realizados ao todo 50 atendimentos, divididos em dois momentos. Nas primeiras 25 sessões, utilizou-se da aplicação da toxina Botulínica A nos músculos espásticos do MSE: bíceps braquial, flexor radial e ulnar, superficial e profundo do carpo e dedos, como também no adutor do polegar, associada à cinesioterapia que consistiu em:

- Exercícios de cintura escapular, com técnicas de Facilitação Muscular Proprioceptiva (FNP), enfatizando póstero-depressão, com o objetivo de inibir o padrão apresentado de MSE em rotação interna e ântero-elevação.
- Exercícios de membro superior, aplicando-se o método neuroevolutivo, com uso de bolas suíças, bastões e cones, estimulando-se a execução de movimentos funcionais, necessários à realização das atividades da vida diária (AVD's).

- Exercícios de tronco, utilizando método neuroevolutivo e bola suíça, para flexoextensão, inclinação lateral e rotação, além de exercícios de plano frontal com rotação externa, objetivando aumento da amplitude de movimento do MSE.

Nas últimas 25 sessões, foi acrescido ao tratamento proposto a Estimulação Nervosa TransCutânea (TENS) Convencional, sendo modulada em uma freqüência de $75 \mathrm{~Hz}$, com intervalo de tempo de $60 \mu \mathrm{s}$, durante 20 minutos por sessão. Um dos eletrodos era disposto na região do trapézio superior esquerdo, ao nível da fossa supra-escapular esquerda, e outro na parte medial do bíceps esquerdo.

Todos os dados foram registrados a cada sessão em ficha catalogada, para posterior análise de resultados. O protocolo de estudo foi claramente explicado à paciente, que assinou termo de consentimento para sua participação neste trabalho.

\section{Resultados}

Os resultados foram obtidos através da análise das avaliações, dos questionários iniciais e finais, das anotações diárias realizadas, associados à evolução percebida pela paciente.

Após dois meses de tratamento, observou-se uma pequena redução da espasticidade (de grau 5 para 4), segundo a Escala de Ashworth, o que facilitou a realização dos exercícios propostos, porém não foi suficiente para a melhora do quadro funcional e álgico da articulação gleno-umeral. Os demais parâmetros do quadro clínico mantiveram-se sem modificações.

Quatro meses após início do tratamento, ao ser realizada a segunda reavaliação, em relação à 
amplitude articular de movimento do MSE, verificouse evolução positiva, conforme goniometria, da flexão $\left(0^{\circ}\right.$ para $\left.93^{\circ}\right)$, hiperextensão $\left(0^{\circ}\right.$ para $\left.33^{\circ}\right)$ e abdução de ombro $\left(0^{\circ}\right.$ para $\left.72^{\circ}\right)$, bem como da extensão de cotovelo $\left(0^{\circ}\right.$ para $\left.150^{\circ}\right)$. A aquisição dessas amplitudes articulares foi relevante, uma vez que permitiu o alcance de um maior nível de independência para a paciente, durante suas atividades da vida diária.
Quanto aos espasmos, segundo escala de Penn, observou-se queda nos escores de 4 para 0 , ou seja, a paciente não mais apresentava espasmos musculares. Na perimetria do ombro, verificou-se um ganho de $2 \mathrm{~cm}$.

Quanto à dor, de acordo com a Escala Analógica Visual, os valores iniciais e finais relatados pela paciente, verificados no Quadro 1, comprovaram acentuada diminuição deste sintoma.

\section{INICIAL}

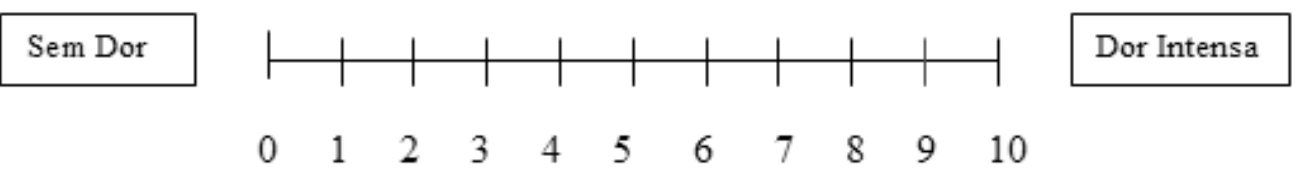

FINAL
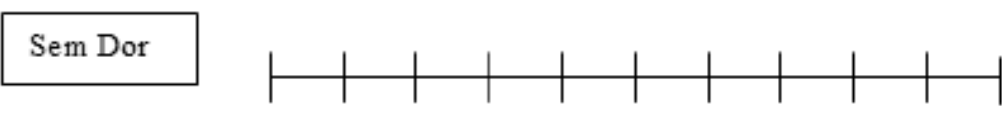

Dor Intensa

$\begin{array}{lllllllllll}0 & 1 & 2 & 3 & 4 & 5 & 6 & 7 & 8 & 9 & 10\end{array}$

Quadro 1. Valores Iniciais e Finais Mensurados pela Escala Analógica Visual de Dor

\section{Discussão}

$\mathrm{O}$ acidente vascular cerebral pode comprometer os princípios biomecânicos normais e a estabilidade do complexo do ombro, devido à perda do controle motor e do desenvolvimento de padrões anormais de movimento. Secundariamente, ocorrem alterações em tecidos moles e desalinhamento da articulação gleno-umeral. Aincidência de ombro doloroso prevalece entre 34 a $85 \%$ dos pacientes, independente de idade e sexo, ocorrendo geralmente na segunda semana após o acidente vascular (KLOTZ et al., 2006).

Dentre as causas mais comuns da síndrome dolorosa, encontram-se a espasticidade (VUAGNAT; CHANTRAINE, 2003), lesões de partes moles (YU, 2003), capsulite adesiva (JÜRGEL et al., 2005), subluxação (PACI; NANNETTI; RINALDI, 2005) e síndrome dolorosa miofascial (SILVA; RIBERTO; BATTISTELL, 2000). No presente relato, a espasticidade pós- AVC foi considerada o fator etiológico responsável pelo desenvolvimento da síndrome, e verificou-se um quadro clínico semelhante ao descrito na literatura: a dor no ombro e perda progressiva de ADM (HORN, 2003), limitação para rotação externa do braço, seguida de dificuldade de abdução e flexão, além de dor à palpação nos pontos de inserção tendínea da cabeça longa dos músculos bíceps braquial, supraespinhoso, infraespinhoso, redondo, grande dorsal, rombóide, trapézio e bainha dos músculos rotadores do ombro (GAL et al, 2001). 
A busca incessante de uma terapêutica antinociceptiva pode ser considerada como um desafio para os profissionais de saúde, uma vez que as várias alternativas para combatê-la nem sempre são eficazes. O arsenal analgésico oficial é representado por recursos terapêuticos alopáticos, homeopáticos, fitoterápicos e neurocirúrgicos. Apesar dos avanços na farmacocinética e na farmacodinâmica dos agentes antiálgicos, sua toxicidade reconhecidamente elevada é determinante de resultados clínicos conflitantes, em função da necessidade de associações e interações medicamentosas, sobretudo na dor crônica em razão de sua bioplasticidade, e relação com quadros clínicos de ansiedade e depressão, que reduzem a qualidade de vida do paciente (VALE, 2006).

A TENS age sobre as fibras nervosas aferentes como um estímulo diferencial que "concorre" com a transmissão do impulso doloroso. Ativa as células da substância gelatinosa, por promover uma modulação inibitória segmentar, e, no sistema nervoso central, estimula a liberação de endorfinas, endomorfinas e encefalinas. A ativação do sistema analgésico central resulta numa diminuição ou bloqueio da percepção central à dor (TRIBIOLI, 2003). Neste estudo, foi verificada uma melhora expressiva nos níveis de dor, com redução em até seis pontos, conforme a EAV, o que sugere ter sido a diminuição da dor o ponto fundamental para melhora expressiva do quadro clínico da paciente envolvida.

Vários autores têm implementado o uso da TBA no tratamento da espasticidade (HORN et al., 2003; FRAGOSO; Araújo; Veloso, 2004; MAKI, 2005), pois ela atua na junção neuromuscular e bloqueia a liberação do neurotransmissor acetilcolina em nível pré-sináptico, provocando paresia muscular transitória (TEIVE; zonta; kumagai,1998). O uso da toxina botulínica tem sua maior indicação na promoção do relaxamento muscular localizado na musculatura mais distal, o que resulta na diminuição da dor de origem espástica e/ou dos espasmos, facilitando o trabalho fisioterapêutico e melhorando as expectativas quanto à recuperação motora dos pacientes com ombro doloroso (PONTES, et al, 2000).

A fisioterapia é uma ciência aplicada, e seu objeto de estudo é o movimento humano, em todas suas formas de expressão e potencialidades, quer nas suas alterações patológicas, quer nas suas repercussões psíquicas e orgânicas, com os objetivos de preservar, manter, desenvolver ou restaurar a integridade de órgãos, sistemas ou funções (REBELLATO, 1999). É de relevante importância sua aplicação na fase crônica do ombro doloroso, para prover o restabelecimento dos movimentos, desaparecimento da dor e a volta da potência muscular (FELLET et al, 2000).

Os resultados de uma determinada técnica ou tratamento são percebidos pela melhora da disfunção presente, portanto a mobilização do ombro foi observada fase a fase, e as diferenças mais significativas ocorreram durante o segundo momento do tratamento, ou seja, após quatro meses de tratamento, quando as três modalidades terapêuticas propostas foram associadas em conjunto. Houve considerável aumento do movimento passivo do MSE e maior estabilidade articular, redução da espasticidade e desaparecimento dos espasmos apresentados inicialmente, o que melhorou as perspectivas em relação à recuperação motora. Após o tratamento fisioterapêutico, observou-se acentuada evolução da dor no ombro e, a paciente relatou maior segurança para realizar as AVD's, além de motivação para continuar o tratamento, com conseqüente aumento da sua qualidade de vida.

\section{Conclusão}

A impossibilidade de generalização dos resultados obtidos com o estudo de caso constitui limitação deste tipo de delineamento (GIL, 2002). Com base nas considerações apresentadas, sugere-se o desenvolvimento de novas investigações, no sentido de aprofundar o referido tema, visando à consolidação de estratégias terapêuticas voltadas às necessidades específicas dos pacientes. 
Os resultados deste estudo confirmam a importância da toxina botulínica A e da fisioterapia no tratamento da síndrome do ombro doloroso pósAVC. A TENS com seu efeito analgésico, aliado ao relaxamento muscular provocado pela toxina botulínica, reduziu o quadro álgico apresentado, e, associada à cinesioterapia, permitiu um aumento da amplitude de movimento articular e da motricidade ativa e passiva, facilitou o trabalho fisioterapêutico e melhorou as perspectivas quanto à recuperação motora, promovendo, assim, uma reintegração da paciente na execução das atividades de vida diária.

\section{Referências}

ACEVEDO, J.C.; PARDO, J.L; JIMENEZ, E; BOGOT, S. de. Secuelas espasticas de lãs enfermidades neurológicas, Acta Médica Colombiana, v. 25, mar./abr., p.75-83, 2000. Disponível em: <http://bases.bireme.br/cgi-bin/ wxislind.exe/iah/online/? Is is Script=iah/ iah.xis\&src=google \&base $=$ LILACS\&lang $=p \&$ nextAction $=$ lnk\&exprSearch=358434\&indexSearch=ID. $>$.

Acesso em: 15 out. 2006.

FELLET, A. J.; SCOTTON, A.S.; FRAGA, R. O.; ZAGUETO, Z. Ombro Doloroso. Revista Brasileira de Medicina, São Paulo, v. 57, p.157-7, 2000 . Disponível em: < h t t p :// w w w. cibersaude.com.br/ revistas.asp?fase $=$ r003\&id_materia $=601>$. Acesso em: 14 ago 2006.

FERNANDES, M.; CARVALHO, L.; PRADO, G. Órtese elétrica funcional usada no membro inferior parético melhora a qualidade de vida em pacientes com acidente vascular encefálico. Arquivos de neuro-psiquiatria, São Paulo, vl.64, n.1, 2006. Disponível em:< http:// www.scielo.br/scielo.php?script=sci_arttext\&pid=S0004$282 X 2006000100005 \& \operatorname{lng}=$ pt\&nrm=iso $>$. Acesso em : 23 de abr. de 2006.

FRAGOSO, Y. D.; ARAUJO, M. G.; VELOSO, S. R. R. Injeção de toxina botulínica A para tratamento do ombro doloroso do hemiplégico. Reabilitar, São Paulo, v.6, n.25, p. 9-13, out./dez. 2004. Disponível em: < http:// bases.bireme.br/cgi-bin/wxislind.exe/iah/online/

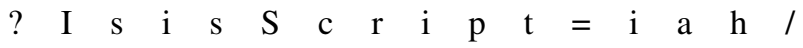
iah. $x$ is $\& s r c=$ google $\&$ base $=$ LILACS\&lang=p\&nextAction= lnk\&exprSearch=413815\&indexSearch=ID >.

Acesso em: 10 set 2006.
GAL, P.; TEIXEIRA, M.; BERGEL, R.; TEIXEIRA, W. Dor torácica. Revista de Medicina, São Paulo, v. 80,n.2, p.:34150, 2001. Disponível em: < http://bases.bireme.br/cgi-bin/ wxislind.exe/iah/online/? Isis Script=iah/ iah.xis\&src=google\&base $=$ LILACS\&lang=p\&nextAction= lnk\&exprSearch=347962\&indexSearch=ID $>$.

Acesso em: 14 ago 2006.

GIL, A. C. Como elaborar projetos de pesquisa. 4.ed. São Paulo: Atlas, 2002.

HOLANDA, M.; FILIZOLA, R. G.; COSTA, M. J. C.; ANDRADE, E. M. F.; SILVA, J. A.G. Avaliação antropométrica em pacientes diabéticos com acidente vascular cerebral isquêmico. Arquivos de neuropsiquiatria, São Paulo, v.64, n.1, 2006. Disponível em: $<$ http://www.scielo.br/scielo.php?script=sci_

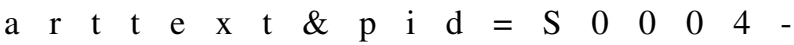
$282 X 2006000100004 \& \operatorname{lng}=$ pt $\& n r m=i s o>$. Acesso em: 23 mar 2006.

HORN, A. I.; FONTES, S. V.; CARVALHO, S. M. R.; SILVADO, R. ${ }^{a}$ B.; BARBOSA, P. M. K.; DURIGAN,J.A.; ATTALHAH, A. N.;FUKUJIMA, M. M.; PRADO, G. F. Cinesioterapia previne Ombro Doloroso em Pacientes Hemiplégicos/Paréticos na fase subaguda do Acidente Vascular Encefálico. Arquivos de neuro-psiquiatria, São Paulo, v. 61, n. 3B, p.768-771, 2003. Disponível em: < http:/ /www.scielo.br/scielo.php?script=sci_arttext\&pid=S0004282X2003000500012\&lng=en\&nrm=iso $>$. Acesso em: 23 ago 2006.

JÜRGEL, J.; RANNAMA, L.; GAPEYEVA, H.; ERELINE, J.; KOLTS, I.; PÄÄSUKE, M.. Shoulder function in patients with frozen shoulder before and after 4-week rehabilitation. Medicina, Estonia, 41 N. 1,p. 30-38, 2005. Disponível em: <http://medicina.kmu.lt/0501/050105e.htm>. Acesso em: 14 ago 2006.

KLOTZ, T.; BORGES, H. C.; MONTEIRO, V. V.; CHAMLIAN, T. R.; MASIERO, D. Tratamento fisioterapêutico do ombro doloroso de pacientes hemiplégicos por acidente vascular encefálico - Revisão da Literatura. Acta Fisiátrica, São Paulo, v.13, n. 1, p. 1216, 2006. Disponível em: <http://www.actafisiatrica.org.br/ v1\%5Ccontrole/secure/Arquivos/AnexosArtigos/ 44F683 A 84163 B 3523 AFE 57 C 2E008 BC 8C/ editoracao_vl_13_n_01_12-16_color.pdf $>$. Acesso em: 14 ago 2006.

LISINSKI, P.; GRABARCZYK, G. Aspects of physiotherapy in treatment of shoulder joint pain. Chirrugia narzadów ruchu ortopedia polska, Warszawa , Polônia, v. 70, n. 4, p. 295-9, 2005. Disponível em: < http:/ /www.ncbi.nlm.nih.gov/pubmed/16521532 >. Acesso em: 23 set 2006. 
LUTTI, M. N.; VIEIRA, J. L.; EICKHOFF, D. R.; CARLI, D.; CARVALHO, M. A. de. Analgesia Controlada pelo Paciente com Fentanil e Sufentanil no Pós-Operatório de Reconstrução de Ligamentos do Joelho:Estudo Comparativo. Revista Brasileira de Anestesiologia, Campinas, vl. 52, n. 2, p.166-74, mar/abr 2002. Disponível em: $<$ http://www.scielo.br/ scielo.php?script $=$ sci_arttext \& pid=S 0034 70942002000200004 >. Acesso em: 20 ago 2006.

MAKI, T. Toxina botulínica tipo A e estimulação elétrica funcional no membro superior de pacientes crônicos pósacidente vascular cerebral. 2005. Dissertação (Mestrado em Ciências Medicas) - Faculdade de Ciências Médicas. Campinas: Universidade Estadual de Campinas.

MACHADO, ÂNGELO. Neuroanatomia Funcional. São Paulo: Atheneu, 2000.

MEYTHALER, J. M. Consept of spastic hypertonia. Physical medicine and rehabilitation clinics of North America, Alabama, phys med rehabil clin n am, v. 12, n. 4, p 725-32, 2001. Disponível em: <http:// w w w. n c bi.n $1 \mathrm{~m}$. ni h.gov/e n t r e z/ query.fcgi? cmd $=$ Retrieve $\& d b=$ pubmed $\&$ dopt $=$ Abstract\&list_uids $=11723862 \&$ query_hl $=3>$. Acesso em :02 jul 2006.

MUSSE, C.A. I.; RIEDER,, C. R.; PICON,,P.D.; AMARAL, K. M. Protocolo clínico e diretrizes terapêuticas: Espasticidade Focal Disfuncional, Toxina Tipo A de Clostridium botulinum. In: PICON, P. D.; BELTRAME, A. (Ed.) Protocolo Clínico e Diretrizes Terapêuticas: medicamentos excepcionais. Brasil: Ministério da Saúde, 2002, p. 341-348.

O’SUlliVAN, S. B; SCHMITZ, T. J. Fisioterapia Avaliação e Tratamento. 2 ed.São Paulo: Manole, 2004.

PACI, M.; NANNETTI, L.; RINALDI, L. Glenohumeral subluxation in hemiplegia: An overview. Journal of rehabilitation research and development, Washington, v. 42, n. 4, p. 557-68, 2005. Disponível em : <http:// $\begin{array}{llllllllllllllll}6 & 4 & . & 2 & 3 & 3 & . & 1 & 6 & 9 & . & 1 & 0 & 4 & /\end{array}$ search?q=cache:jKa5pf8gI6EJ:www.rehab.research.va.gov/ jour/05/42/4/paci.html+Glenohumeral+subluxation+in+ h e mi plegia : + A n + overvie w \& hl l=pt BR\&ct=clnk\&cd=1\&gl=br $>$. Acesso em: 22 ago. 2006.

PÓO, P.; LÓPEZ-CASAS, J.; GÁLVAN-MANSO, M.; AQUINO-FARIÑA, L.; TERRICABRAS-CAROL, L.;CAMPISTOL, J. Botulinum toxin type A: indications and results. Revista de neurologia, Barcelona, v.37, n.1, p.74-80, 2003. Disponível em: <http://www.revneurol.com/ sec/resumen.php?or=pubmed\&id=2003221 $>$. Acesso em: 22 set 2006.
PONTES, L. S.; FONTES, S.; BOTELHO, L.; FUKUJIMA, M. Toxina Botulínica Tipo A em Pacientes com Toxina Botulínica Tipo A em Pacientes com Hemiplegia e/ou Hemiparesia. Revista Neurociências, São Paulo, v.8, n. 3, dez. 2000. Disponível em: <http://www.unifesp.br/dneuro/ neurociencias/Neurociencias\%2008-3.pdf $>$. Acesso em: 21 set 2006.

REBELLATO, J.R. Fisioterapia no Brasil. 2.ed. São Paulo: Manole, 1999.

SENEVIRATNE, C.; THEN, K. L.; REIMER, M. Post-stroke shoulder subluxation: a concern for neuroscience nurses. Axone, Calgary, v. 27, p.26-31, 2005. Disponível em: <http:/ /www.ncbi.nlm.nih.gov/pubmed/16259232 >. Acesso em: 14 ago 2006.

SILVA, C.O.; RIBERTO, M.; BATTISTELL, L.R. Avaliação Da dor do ombro em pacientes com acidente vascular cerebral. Acta Fisiatrica, São Paulo, v.7, n. 2, p. 78- 83, 2000. Disponível em: <http://www.actafisiatrica.org.br/ v1\%5Ccontrole/secure/Arquivos/AnexosArtigos/ 0 A 09C 8844 B A 8F0936C 20B D 791130 D 6B 6/ vl_07_n_02_78_83.pdf >. Acesso em: 25 jul 2006.

TEIVE, H.A.; ZONTA, M.; KUMAGAI, Y. Tratamento da espasticidade : uma atualização. Arquivos de neuropsiquiatria, São Paulo, v. 56, n. 4, 1998. Disponível em: $<\mathrm{h} \mathrm{t} \mathrm{t} \mathrm{p} \mathrm{:} \mathrm{/} \mathrm{/} \mathrm{w} \mathrm{w} \mathrm{w.} \mathrm{s} \mathrm{c} \mathrm{i} \mathrm{e} \mathrm{l} \mathrm{o} \mathrm{.} \mathrm{b} \mathrm{r} \mathrm{/}$ scielo.php?script=sci_arttext $\&$ pid=S0004282X1998000500025 >. Acesso em: 28 mar.2005.

TRIBIOLI, R. A. Análise Crítica atual sobre a TENS envolvendo parâmetros de estimulação para o controle da dor.2003. Dissertação (Mestrado em Bioengenharia) Escola de Engenharia de São Carlos, Faculdade de Medicina de Ribeirão Preto, Instituto de Química de São Carlos. Ribeirão Preto: Universidade de São Paulo, 2003.

VALE, N. B. Analgesia adjuvante e alternativa. Revista Brasileira Anestesiologia, Campinas, v. 56, n. 5, 2006. Disponível em: <http://www.scielo.br/ scielo.php?script $=$ sci_arttext $\&$ pid $=$ S $0034-$ $70942006000500012 \& \operatorname{lng}=\mathrm{en} \& \mathrm{nrm}=\mathrm{iso} \& \operatorname{lng}=\mathrm{pt}>$. Acesso em: 12 dez 2006.

VUAGNAT, H.; CHANTRAINE, A. Shoulder pain in hemiplegia revisited: contribution of functional electrical stimulation and other therapies. Journal of Rehabilitation Medicine, Stockholm, v.35, n.2, p.49-54; quiz 56, 2003. Disponível em: < http://www.ncbi.nlm.nih.gov/pubmed/ 12691333 > Acesso em: 22 set 2006. 
YELNIK, A. P.; COLLE, F. M.C.; BONAN, I. V. Treatment of Pain and Limited Movement of the Shoulder in Hemiplegic Patients with Botulinum Toxin A in the Subscapular Muscle. European Neurology, Paris, v. 50, n.2, 2003 . Disponível em: <http://content.karger.com/ $\begin{array}{llllllllllll}P & \mathrm{r} & \mathrm{o} & \mathrm{d} & \mathrm{u} & \mathrm{k} & \mathrm{t} & \mathrm{e} & \mathrm{D} & \mathrm{B} & /\end{array}$ produkte.asp?Aktion $=$ ShowAbstract $\&$ ArtikelNr $=72505 \&$ Ausgabe $=229478 \&$ ProduktNr $=223840>$. Acesso em: 14 ago 2006.
YU, D. Shoulder pain in hemiplegia. Psysical medicine and rehabilitation of North America, Seattle, v. 15, n. 3, p.683-97, 2003. Disponível em: <http:// w w w. n c bi.n l m.n i h.gov/pub m ed/ 15219895?ordinalpos=4\&itool=EntrezSystem2.PEntrez. Pubmed.Pubmed_ResultsPanel.Pubmed_RVDocSum > . Acesso em: 15 jul 2006. 